Archived version from NCDOCKS Institutional Repository http://libres.uncg.edu/ir/asu/

\title{
Appalachỉan
}

B O O NE, NORTH CAROLINA

\section{Is soapy water a viable solution for handwashing in schools?}

\author{
By: Richard D. Rheingans, Shadi Saboori And Alex Mwaki
}

\begin{abstract}
Despite the known health benefi ts of washing hands with soap, global handwashing rates are low. In Nyanza Province, Kenya, a follow-up of 55 pilot primary schools three years after the implementation of a safe water and hygiene intervention revealed that only 2 per cent (one school) provided soap for handwashing on the day of the assessment. After identifying barriers to soap provision, SWASH+ partners piloted a handwashing intervention using powdered soap mixed with water to create soapy water in place of bar soap in 11 schools. The fi rst six months of unannounced visits showed high uptake (10 schools). A one-year follow-up visit revealed a decrease of soapy water use (four schools). This paper discusses the soapy water intervention, initial and follow-up monitoring fi ndings, potential sustainability drivers of handwashing programmes in rural primary schools and next steps.
\end{abstract}

Richard D. Rheingans, Shadi Saboori And Alex Mwaki (2010) "Is soapy water a viable solution for handwashing in schools?" Waterlines 29(4) pp. 329-336 Version of Record Available From www.developmentbookshelf.com 
Three years after a safe water and hygiene intervention only 1 school provided soap for handwashing
ACUTE RESPIRATORY INFECTIONS ARE the leading cause of death in under-fives globally with approximately 2 million children dying from pneumonia each year (UNICEF, 2008). One and a half million people worldwide, the majority of whom are children, die as a result of diarrhoea, the second leading cause of under-five mortality (Prüss-Üstün et al., 2008). A quantitative systematic review of handwashing and the risk of respiratory infections concluded handwashing could decrease the risk of respiratory infections by 16 per cent (Rabie and Curtis, 2006). Another systematic review estimated handwashing with soap could decrease the risk of diarrhoeal diseases by 42-47 per cent (Curtis and Cairncross, 2003).

There is little doubt that washing hands with soap before and after key daily events can dramatically decrease the risk of life-threatening illnesses. As a result, various handwashing programmes are funded every year and implemented worldwide. However, the ability for these programmes to be sustained over time remains a key challenge. Monitoring for sustainability during and after project implementation is essential to help identify potential barriers and change the intervention accordingly.

Shadi Saboori (ssaboor@emory.edu) is a Senior Project Research Coordinator at the Center for Global Safe Water at Emory University, Atlanta, GA, USA. Alex Mwaki is with CARE Kenya and Richard D. Rheingans is at the Hubert Department of Global Health, Rollins School of Public Health, Emory University, Atlanta, Georgia, USA. 


\section{Background of intervention}

The Sustaining and Scaling School Water, Sanitation, and Hygiene Plus Community Impact (SWASH +) project is a five-year (2006-2011) water, sanitation and hygiene applied learning project led by CARE. The SWASH + project activities include a three-year randomized controlled trial (RCT) study testing water, sanitation and hygiene (WASH) interventions in 185 rural primary schools in Nyanza Province, Kenya. Understanding both the key barriers and the drivers determining the sustainability of school WASH interventions is one of the main objectives of the project.

In May 2008, SWASH + partners conducted an assessment of a CARE project in 55 pilot rural primary schools that received a safe water and hygiene intervention in 2005. The purpose was to assess the sustainability of the WASH interventions. The results were shared with implementing partners in order to help them make informed decisions on potential changes in the ongoing RCT study intervention schools. Results revealed that only 2 per cent of the pilot schools provided soap for handwashing on the day of the assessment. The two main barriers to soap provision identified during the sustainability assessment based on interviews with teachers were soap theft (29 per cent) and insufficient or lack of school funds (60 per cent) (Saboori et al., 2010).

Project partners strategized together on potential approaches to address sustainable provision of soap in schools in light of the identified barriers. The approach had to incorporate locally available materials, be cost efficient and discourage soap theft. The project partners decided to pilot use of powdered soap mixed with water in bottles with perforated tops. The hypothesis was that children would be less likely to take the bottles off school grounds, encouraging school management to consistently provide soap for handwashing. The regular presence of soap would in turn encourage pupils to exhibit sustained, improved handwashing behaviour.

The handwashing intervention was piloted and monitored over a six-month period from October 2008 to March 2009 in 11 schools. A follow-up visit was conducted one year later in March 2010. This paper documents the piloting of soapy water as an alternative form of handwashing in 11 schools, and discusses findings, lessons learned and next steps.

\section{Methods}

SWASH + partners selected 11 schools in the former districts of Nyando, Rachuonyo and Suba for the pilot. The prerequisites for selection were twofold. First, the chosen schools were not to be part of 
the original $185 \mathrm{SWASH}+$ schools in the intervention trial, in order to prevent contamination of the larger ongoing impact study. Second, all pilot schools had to have al ready received the Safe Water System (SWS), one of the SWASH + interventions.

The SWS intervention components include provision of locally available water treatment inputs, safe drinking and handwashing water containers and hygiene training. An evaluation completed in 2006 in Homa Bay, Kenya, on the impact of the SWS system on 9 out of 45 pilot schools revealed a 35 per cent decrease in school absenteeism compared with a 5 per cent increase in nine neighbouring comparison schools (O'Reilly et al., 2008). The SWS intervention emphasizes handwashing with soap during key events, and treatment of drinking and handwashing water with a locally available water treatment product prior to use. The handwashing water containers consist of plastic buckets with taps and securely fastening lids, and handwashing stations aretypically placed in a central location near latrines ( $O$ 'Reilly et al., 2008). Treatment of handwashing water is recommended because it has been observed that younger primary schoolchildren sometimes drink from handwashing water containers.

SWASH+field staff gave each of the 11 pilot schools between 6 and $12 \mathrm{lkg}$ packets of a locally available powdered soap. Each school was encouraged to make soapy water solution using the powdered soap and to use locally available bottles to store the soapy water next to handwashing stations provided by the SWASH+ project. SWASH+ field staff recommended the schools use a tablespoon of powdered soap in $500 \mathrm{ml}$ bottles.

Data collection included a questionnaire with an observational section whereSWASH +field staff spent on average 30 minutes during one of the designated classroom break periods to observe and record the number of pupils using soapy water after latrine use. Handwashing water was tested for chlorine residual. Four rounds of monitoring were carried out from October 2008 to March 2009. In the October 2008 and January 2009 rounds, SWASH + field staff only visited 10 out of 11 schools because of logistical limitations. SWASH + field staff reported that all four rounds of the monitoring visits were unannounced. In March 2010, one year after the last monitoring round, an unannounced follow-up visit was conducted in all 11 schools.

\section{Monitoring results}

During both the 2008 to 2009 monitoring visits and the 2010 follow-up visit, the majority of the schools were observed to provide handwashing water for pupil use. Among the schools that provided handwashing water on the day monitored, the presence of soapy water near handwashing containers increased from three schools in 
The proportion of reported powdered soap mixed in water varied from school to school
October 2008 to 10 schools in March 2009. During the 2010 followup visit, 4 out of 11 schools had soapy water available for use (see Figure 1). The proportion of reported powdered soap mixed in water varied from school to school and has been detailed in Table 1.

Table 1. Dilution of soapy water in the trials

\begin{tabular}{lcc}
\hline School Name & $\begin{array}{c}\text { Amount of soap } \\
\text { powder used }\end{array}$ & $\begin{array}{c}\text { Volume of each soapy } \\
\text { water bottle }\end{array}$ \\
\hline Ndori RC & 6 teaspoons & 1 liter \\
Kowire & 2 teaspoons each & $150 \mathrm{ml}$ \\
Ragen AIC & 3 tablespoons per bottle & $500 \mathrm{ml}$ \\
Masogo* & handful & $300 \mathrm{ml}$ \\
Nyatwere & missing & missing \\
Dol Kodera & handful & $300 \mathrm{ml}$ \\
Utajo & 2 spoonfuls & $0.5 \mathrm{liter}$ \\
Kamgere & 2 spoonfuls & $0.5 \mathrm{liter}$ \\
Temo & 2 spoonfuls & $0.5 \mathrm{liter}$ \\
Kamasengere & 2 spoonfuls & $500 \mathrm{ml}$ \\
Wanyama & 3 cupfuls & $500 \mathrm{ml}$ \\
\hline
\end{tabular}

Among the schools that provided handwashing water during the 2008 to 2009 monitoring visits, handwashing water was tested for presence of chlorine residual in the last three rounds. Five out of eight, nine out of ten, and seven out of ten tested positive for chlorine residual, respectively. During the 2010 follow-up visit, four out of the eight tested positive for chlorine residual (see Figure 1).

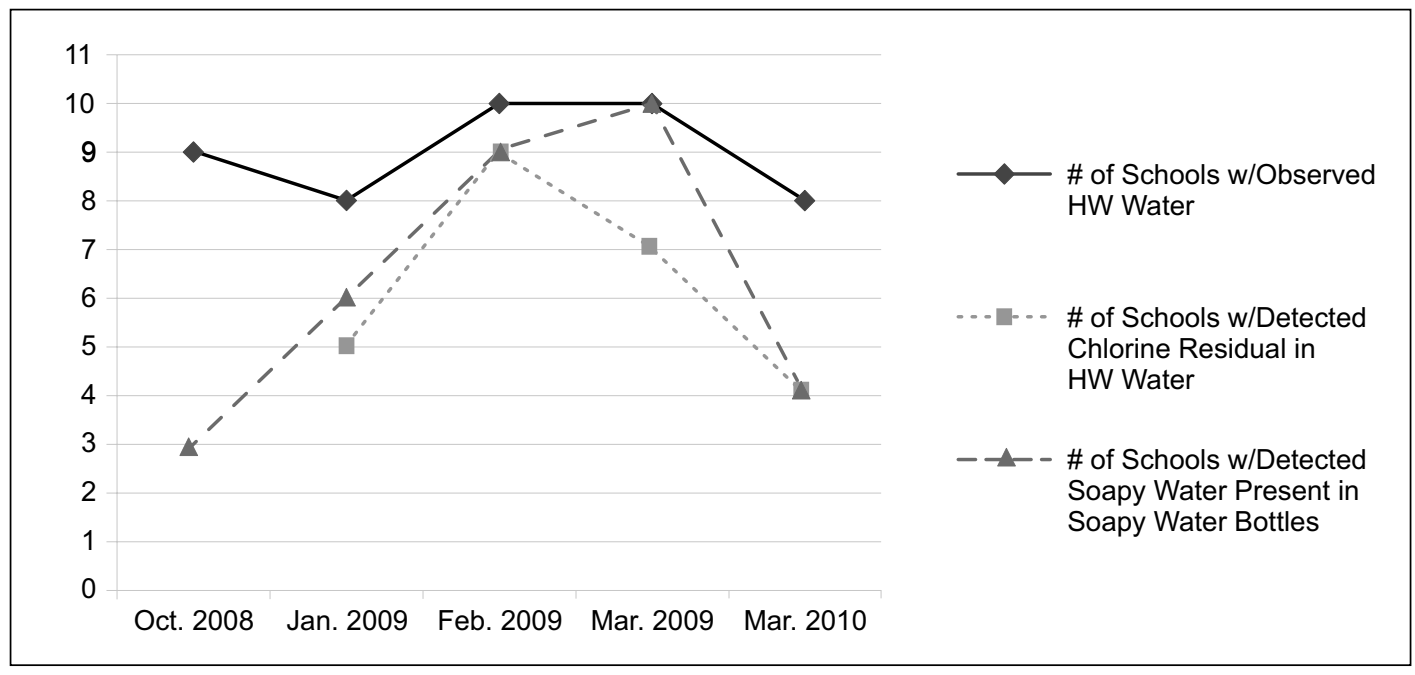

Figure 1. Observed presence of soapy water solution, chlorine residual, and handwashing water in rural primary schools, Nyanza Province, Kenya. 


\section{Observations were} made of pupil handwashing after latrine use and use of soapy water solution
SWASH + staff conducted observations of pupil handwashing after latrine use during designated school break periods. They noted if the pupil also used soapy water solution in the schools that provided soapy water. In the February and March 2009 monitoring visits, only 5 out of 9 schools and 5 out of 10 schools which provided soapy water were observed owing to reported field staff time limitations. The percentage of pupils observed using the latrine and then washing their hands with soapy water increased from 34 per cent in October 2008 to 70 per cent in the final round in March 2009. The January 2009 monitoring round was the highest observed, with 89 per cent. During the 2010 follow-up, of the four schools that provided soapy water on the day of the visit, 50 per cent of pupils were observed washing their hands with soapy water after using the latrines (see Figure 2).

During the 2008 to 2009 monitoring visits, several teachers interviewed suggested the need for 'stronger' or 'more durable' bottles. Most of the schools were reusing plastic soda bottles and noted the bottles were losing their shape as a result of being placed outside in the sun near or on top of handwashing containers. Some interviewees also suggested providing standard sized bottles to make it easier to mix the project-recommended soapy solution (1 tablespoon of powdered soap per 500 millilitres of water). In the 2010 follow-up, six schools reported having some of their soapy water bottles broken and five of those schools reported either replacing all or some of the broken bottles.

During the 2010 follow-up, 10 of the 11 schools reported purchasing powdered soap instead of bar soap or liquid soap in 2009. Reasons reported for purchasing powdered soap over bar soap or liquid soap included easier use (eight schools), longer lasting (six schools), and

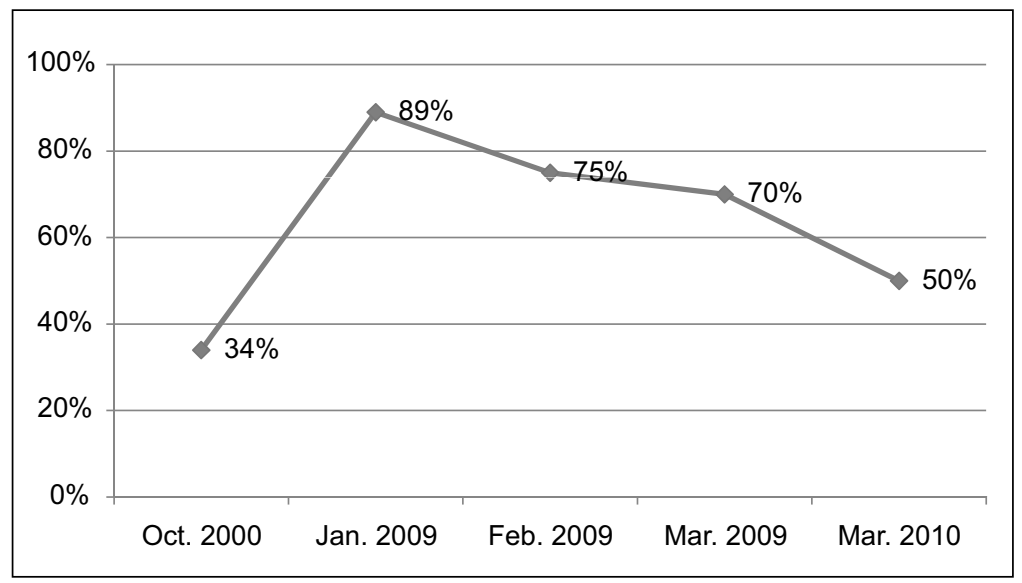

Figure 2. Percentage of pupils observed using soapy water for handwashing after latrine use in rural primary schools, Nyanza Province, Kenya. 
reduced soap theft (six schools). Eight schools reported that the quantity of soap the school was able to provide in the 2009 school year was insufficient to provide pupils with soap on a daily basis, and 10 schools reported that the amount budgeted for soap in 2009 was insufficient.

\section{Discussion and next steps}

One suggested driver for the high uptake of soapy water during the 2008 to 2009 period may be the influence of monitoring visits conducted by the SWASH + field staff. In the 2010 follow-up, the interviewer noted that, in one of the schools that had not provided soapy water on the day of the unannounced monitoring visit, a teacher cut up pieces of bar soap and mixed it with water in a bottle after realizing the purpose of the monitoring visit. A system of regular unannounced monitoring visits to schools by local officials may foster a greater sense of accountability within the school leadership, regardless of the intervention used. Although the monitoring visits were unannounced, it is possible that teachers may have instructed pupils to wash their hands with soapy water upon seeing the arrival of the SWASH + field staff. The authors acknowledge that the direct observations made in this pilot are probably more than what would occur in the absence of an outside observer and likely an overestimate.

2010 follow-up

The March 2010 follow-up results compared with the March 2009 results showed a decrease in the number of schools with soapy water (10 schools compared with 4). One potential reason for the overall decrease could be the one-year lag in monitoring by SWASH + staff. Another could be insufficient funds for soap provision. The majority of schools reported that the amount budgeted and the quantity of soap provided in the 2009 school year was insufficient to provide pupils with soap on a daily basis. Although soap theft, which was one of the two identified barriers to soap provision in rural primary schools, was reduced as a result of the soapy water intervention, sufficient funding of soap remains a barrier regardless of the handwashing method implemented. Additionally, between March 2009 and March 2010 there was a 29 per cent decrease in the proportion of students observed using soapy water after visiting latrines in schools where soapy water was present. This trend may potentially be attributed to inadequate funds preventing regular provision of soap. Intermittent breaks in soap provision are likely to decrease the chances of adopting regular handwashing habits.

Currently, the Kenyan Ministry of Education allocates 10 Kenyan Shillings (US\$0.13) per primary school pupil per year for electricity, water and sanitation (Sawamura and Sifuna, 2008). The amount is 
For sustainability allocations

for recurrent

costs must be

planned prior to

implementation

Repurchase rates and preference

for soapy water

suggests it may be

a more sustainable

method of

handwashing insufficient for the maintenance and upkeep of school WASH facilities and purchase of consumables such as soap. To foster sustainability of WASH facilities, allocations for recurrent costs must be planned prior to implementation.

All but one of the pilot schools reported preference towards powdered soap over bar or liquid soap in 2009. A majority of school interviewees found powdered soap cheaper and easier to use. A majority of schools reported that theft of soap reduced. Additionally, 10 of the 11 schools reported repurchasing powdered soap over bar or liquid soap during the 2009 school year. The higher rate of repurchase and preference for soapy water suggests that it may be a more sustainable method of handwashing than the current alternatives, but this was not directly tested in this pilot study. The authors were unable to find similar studies using soapy water for handwashing. In a 2009 study, improvised soap dispensers were recommended for Kenyan schools in order to prevent wastage of soap on school grounds (Water and Sanitation Program, 2009).

Given the small sample size, lack of control schools, and variability in the number of pilot schools visited and observed between monitoring rounds, SWASH + partners determined the need for further study. A 2010 study of 60 schools, including controls, has been planned. The study will include the provision of durable and standardized locally available bottles for soapy water provision and will measure use, feasibility and acceptability over two to three school terms. A 2010 study looking at the use of alcohol hand-gels versus unmedicated soap and water for handwashing among nurses found a significant increase in skin irritation and dryness using the latter (Boyce et al., 2000). The planned study will identify any potential side-effects observed among pupils using soapy water for handwashing.

The CARE-recommended powdered soap-to-water mixture was a chosen starting point for this pilot. A separate study in 2010 will compare the effectiveness of handwashing with soapy water, bar soap and water al one in reducing faecal contamination on the hands of pupils in four selected schools. The study will use a laboratory recommended dosage for creating the soapy water mixture.

Assuming adequate funds are allocated to purchase soap, initial results of using soapy water in rural primary schools as a form of soap provision for handwashing are promising.

\section{References}

Boyce, J.M., Kelliher, S. and Vallande, N. (2000) 'Skin irritation and dryness associated with two hand-hygiene regimens: Soap-and-water handwashing versus hand antisepsis with an alcoholic hand gel', Infection Control and Hospital Epidemiology 21: 442-8. 
Curtis, V. and Cairncross, S. (2003) 'Effect of washing hands with soap on diarrhoea risk in the community: a systematic review', Lancet Infectious Diseases 3: $275-81$.

O'Reilly, C.E., Freeman, M.C., Ravani, M., Migele, J., Mwaki, A., Ayalo, M., Ombeki, S., Hoekstra, R.M. and Quick, R. (2008) 'The impact of a school-based safe water and hygiene programme on knowledge and practices of students and their parents: Nyanza Province, western Kenya, 2006', Epidemiology \& Infection 136: 80-91.

Prüss-Üstün, A., Bos, R., Gore, F. and Bartram, J. (2008) Safer Water, Better Health: Costs, Benefits and Sustainability of Interventions to Protect and Promote Health, World Health Organization, Geneva.

Rabie, T. and Curtis, V. (2006) 'Handwashing and risk of respiratory infections: a quantitative systematic review', Tropical Medicine and International Health 11: 258-67.

Saboori, S., M waki, A., Porter, S.E., Okech, B.O., Freeman, M.C. and Rheingans, R.D. (2010) Sustaining School Hand Washing and Water Treatment Programs: Lessons Learned and to be Learned, The Center for Global Safe Water at Emory University, Atlanta, GA.

Sawamura, N. and Sifuna, D.N. (2008) 'Universalizing primary education in Kenya: Is it beneficial and sustainable?' Journal of International Cooperation in Education 11: 103-18.

UNICEF (2008) The State of the World's Children 2008, UNICEF, New York.

Water and Sanitation Program (2009) Are Your Hands Clean Enough? Study Findings on Handwashing with Soap Behaviour in Kenya, WSP, UNICEF, Nairobi, Republic of Kenya. 